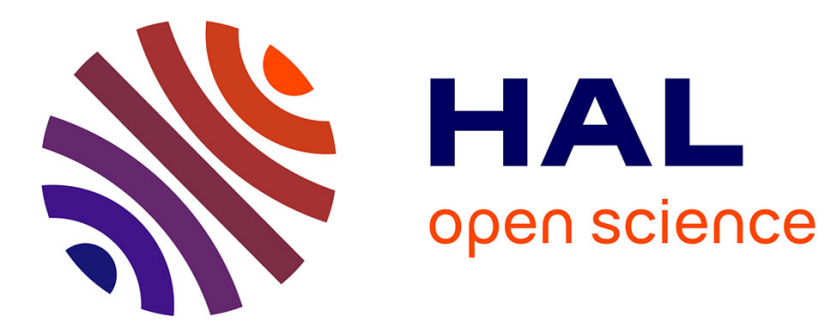

\title{
Macro-modelling of Lightning strokes on Aircraft
}

\author{
B. Michielsen, F. Issac, P. Aguilera, D. Prost
}

\section{To cite this version:}

B. Michielsen, F. Issac, P. Aguilera, D. Prost. Macro-modelling of Lightning strokes on Aircraft. IEEE 2014 XXXIth URSI General Assembly and Scientific Symposium (URSI GASS), Aug 2014, BEIJING,

China. hal-01071575

\section{HAL Id: hal-01071575 \\ https://hal-onera.archives-ouvertes.fr/hal-01071575}

Submitted on 6 Oct 2014

HAL is a multi-disciplinary open access archive for the deposit and dissemination of scientific research documents, whether they are published or not. The documents may come from teaching and research institutions in France or abroad, or from public or private research centers.
L'archive ouverte pluridisciplinaire HAL, est destinée au dépôt et à la diffusion de documents scientifiques de niveau recherche, publiés ou non, émanant des établissements d'enseignement et de recherche français ou étrangers, des laboratoires publics ou privés. 


\title{
Macro-modelling of Lightning strokes on Aircraft
}

\author{
B. Michielsen ${ }^{* 1}$, F. Issac ${ }^{1}$, P. Aguilera ${ }^{2}$ and D. Prost ${ }^{1}$ \\ 1 Onera, Toulouse, France, \\ Bastiaan.Michielsen@Onera.fr, Francois.Issac@Onera.fr, Daniel.Prost@Onera.fr \\ 2 Snecma, Villaroche, France, Paula.Aguilera@Snecma.fr
}

\begin{abstract}
Lightning phenomena are relatively slow electromagnetic processes and can be modelled in quasi-static approximation even on rather large structures like aeroplanes. Modelling the interaction of lightning currents with electronic equipment in an aircraft is a great challenge because of the geometrical complexity. Using universal methods like finite difference or finite element techniques on a complete mesh of the entire configuration is not an option in a design stage where many iterations are required. In this paper, we describe a macro-modelling technique which accounts for the geometrical detail while taking benefit from the quasi-static nature of the phenomena. We show that with these techniques rapid time domain (or frequency domain) simulations on complete lightning configurations can be done giving results very close to the results of exhaustive universal modelling and measurement.
\end{abstract}

\section{Introduction}

In the analysis and optimisation of lightning protection in aircraft, one needs to do many iterations on complex interaction geometries. Universal modelling tools accounting for complex geometries often suffer from extreme computation times because the stability of universal discretisation methods requires a very small time step corresponding to the finest structure in the complete mesh.

In this paper, we propose a macro-modelling technique which, from the start, acknowledges the quasi-static nature of the phenomena and represents the entire configuration in a generalised Hamiltonian model consisting of model components for storing electric and magnetic energy as well as for various loss mechanisms (see [1]). The relations defining these model components are constructed from current and charge distributions defined on the fine mesh of the interaction configuration. Using the mesh-intrinsic Laplace operator, we construct a limited set of vector fields on the mesh which are suitable candidates to span a space of current (and charge) distributions. The model-component coefficients are then computed as the inductance, resistance and capacitance coefficients of these distributions and their divergences, respectively. Finally, the resulting relations are subject to charge conservation constraints in the form of Kirchhoff interconnect relations. This scheme leads to a network model with relatively few degrees of freedom.

The strategy can be compared to PEEC modelling (see [2]), however, we emphasise the fact that the coefficients of the model components depend on the choice of the current distributions. The current distributions we are interested in are specific for lightning phenomena and our model construction starts with establishing a convenient set of current distributions adapted to a chosen excitation. In this paper, therefore, we do not start with the Maxwell equations and a discretisation conforming to geometrical requirements. Instead, we put a model on the foreground based on global current distributions and then compute the coefficients for the electromagnetic interaction using Maxwell theory. The numerical computations are indeed done by expanding the chosen current distributions in conventional finite element spaces. This method can also be compared to hierarchical techniques in integral equation modelling ([3]) and indeed we use techniques, previously applied in that context, to find partitionings of the interaction configuration (see [4]). However, our purpose is not to define a universal model and, therefore, we formulate our model on a single level in such a hierarchy which provides us with the pertinent degrees of freedom to obtain a good correspondence with only very specific physical phenomena.

\section{Generalised Hamiltonian models}

The generic, linear, port-Hamiltonian model constituent we use is of this form

$$
\begin{aligned}
M \partial_{t} \psi & =d H \phi+C u \\
v & =C^{t} \psi
\end{aligned}
$$

where $d H$ maps "momentum" coordinates on the state space (the definition of which remains implicit) to forces and $M$ is a kind of inverse "phenomenological" coefficient. The $u$ are referred to as "controls" and the $v$ as "responses".

In a first instance we construct a direct sum model with

$$
X=\oplus_{p=1}^{n} X_{n}
$$

for $X=M, \psi, H, \phi, C, u, v$.

To transform the juxtaposition of the sub models into a single evolution equation, we have to add compatibility constraints. In our electromagnetic model these compatibility constraints are derived from charge conservation.

The general scheme of the previous section will now be made explicit for an electromagnetic interactions. The sub models we use in an electromagnetic interaction configurations can themselves be macro models. For example, a sub model may be a multi-conductor transmission line of a certain length for which the controls and responses are voltages and/or currents a the terminal ports. Another sub model may be some mechanic part which has already been given a 
macro-model. We can, however, identify three elementary models for resistive loss, magnetic energy and electric energy, from which all the models are built.

Every elementary model is represented by a branch with two end nodes. On the branches we define current and voltage functions, $I$ resp. $V$ and on the nodes we define charge and potential functions, $q$ resp. $\varphi$. The elementary models are of three types: a resistive, an inductive and a capacitive model

$$
V=R I \quad L \partial_{t} I=V \quad C \partial_{t} V=I
$$

The compatibility constraints are derived from charge conservation on the interconnection graph of the elementary models. In the space of functions on this graph we have a differential operator $d$ and its adjoint $\delta$. These operators restricted to functions which are constant on the branches of the graph reduce to matrix operators on the values and can be identified with the branch-node incidence matrix and its transpose. The charge conservation relation is then expressed per node as a constraint on the current divergence

$$
\delta I=0
$$

and because of $\delta \circ \delta=0$ we can satisfy this equation by using

$$
I=\delta G
$$

for some $G$ defined on the loops of the graph. There is no unique $G$ for a given $I$, but we can transform $G$ into a function on the branches of the graph by an operator $\sigma$ and require for $G=\sigma V$

$$
d V=0 \Leftarrow V=d \varphi
$$

such that we get a Laplace equation

$$
\delta \sigma d \varphi=I
$$

and, due to the invertibility of the Laplacian,

$$
\varphi=[\delta \sigma d]^{-1} I
$$

Finally, we obtain the two constraints $I \in \operatorname{ker}(\delta)$ and $V \in \operatorname{im}(d)$, which are Kirchhoff's relations.
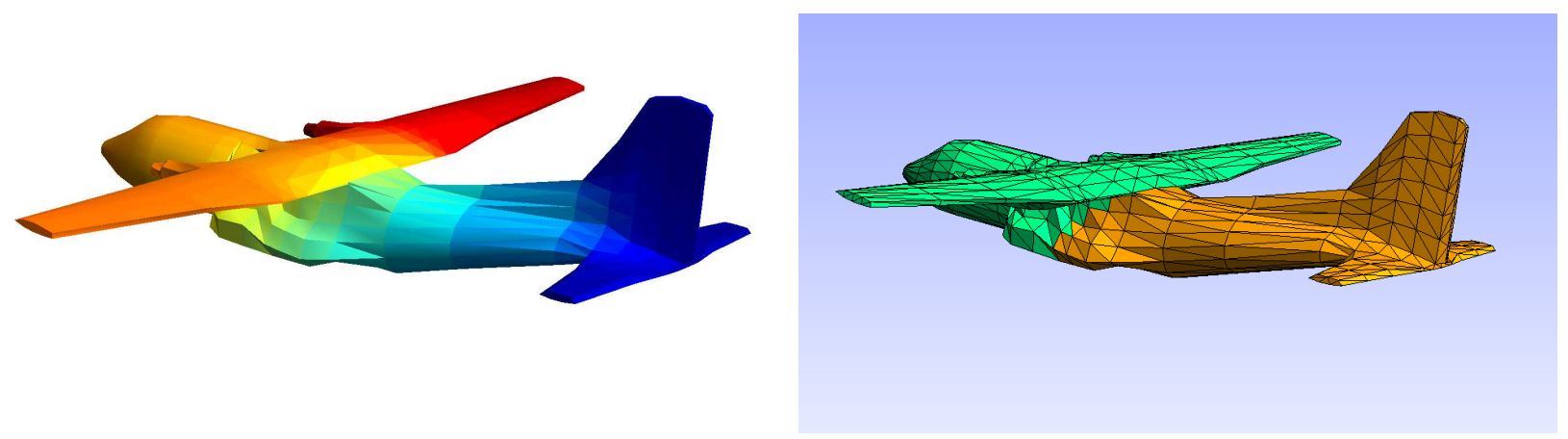

Fig. 1 An eigenfunction of an intrinsic Laplace operator and its associated domain decomposition

\section{Constructing the model coefficients}

In the preceding sections, we have presented a framework for macro-modelling using electromagnetic models with inductive, capacitive and resistive components. We obtained an evolution equation by combining the model components such that charge conservation is warranted. Now, we do not want to measure the elementary model coefficients but we want to compute them numerically using a geometrical model in the form of a mesh. An essential observation, here, is that coefficients like resistance, inductance and capacitance are not defined by the geometry and physical constitutive coefficients alone but they apply to a current/charge distribution in the physical configuration. The clue to computing the coefficients of the model is to construct appropriate current distributions on the geometry. Once we have these, let's call them $j_{p}$ with $p=1, \ldots, N$, we can compute the coefficients with

$$
\begin{aligned}
L_{p q} & =\int j_{p} \cdot A\left(j_{q}\right)=\int j_{p}(x) \cdot \int G(x, y) j_{q} \\
C_{p q} & =\int \rho_{p} \cdot \Phi\left(\rho_{q}\right)=\int \rho_{p}(x) \cdot \int G(x, y) \rho_{q} \\
R_{p q} & =\int j_{p} \cdot r j_{q}
\end{aligned}
$$


where $r$ is the resistance density and $\rho_{q}=\operatorname{div} j_{q}$.

The heart of the macro-modelling is therefore the choice of the current distributions. We can obtain these by constructing a macro finite element space as a particular subspace of a conventional finite element space based on the geometry determined triangulation of the interaction configuration. A Laplacian operator associated to a 1-skeleton of the simplicial complex dual to the triangulation is used on two levels of the construction. It's spectral resolution is used to define a binary tree of subdivisions (see Fig. 1) and solutions of specific Dirichlet boundary value problems on the subdivisions and/or on the complete geometry are used to construct, divergence-free or gradient type current distributions. An example of a global current distribution is shown on Fig. 2. This current distribution is computed from a mesh Laplacian for imposed current injection and current extraction points. Such current distributions can be computed extremely rapidly by a simple relaxation method. To account for proximity effects which come into play

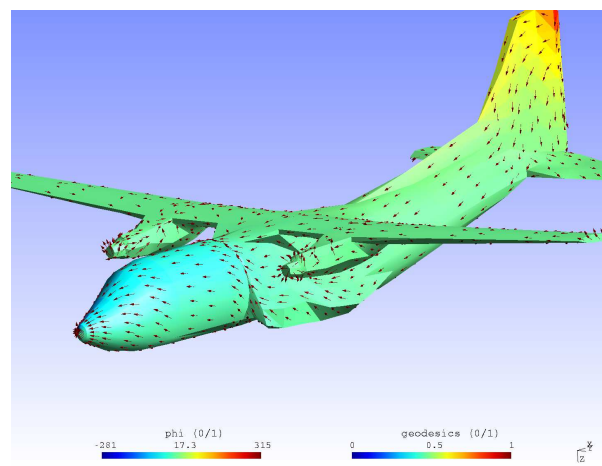

Fig. 2 Global lightning current from rear to nose.

when the inductive interaction model on the micro-level becomes more important than the purely resistive model, we propose a co-simulation with two quasi-static models. This relies on two sets of current distributions, $J_{0}$ and $J_{1}$, on the chosen partitions of the interaction configuration. The distributions $J_{0}$ correspond to the purely static phenomena and are determined by resistances and solutions of the Laplace equation. The distributions $J_{1}$ account for inductive interactions and are constructed such as to include the proximity effects, i.e., currents concentrate where self-induction is minimal or where the proximity of other currents of possibly opposed polarity can diminish impedance.

In the time-evolution of the state, the co-simulation amounts to computing the state's time derivative by applying a low-pass filter and its complement to the state vector and using the $J_{0}$-based model coefficients respectively the $J_{1}$-based model coefficients to advance the respective state constituents.

\section{Example}

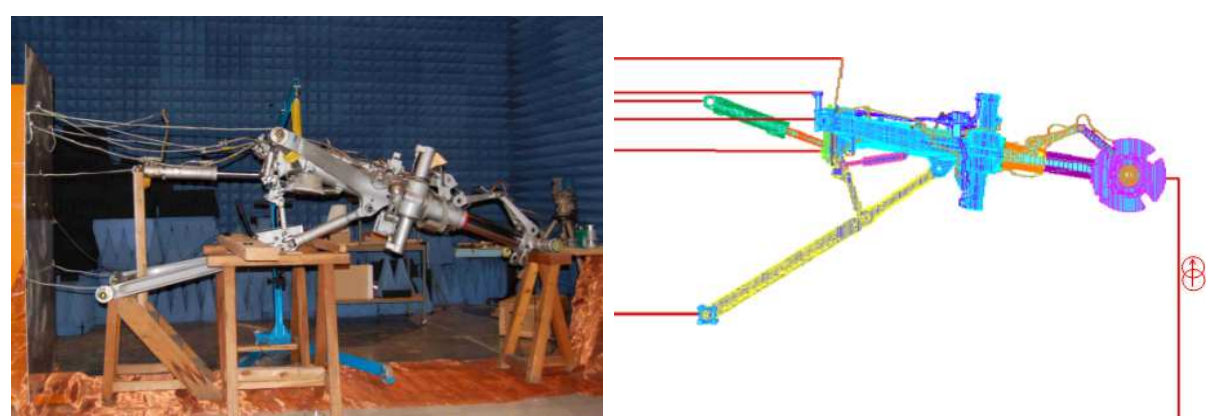

Fig. 3 Landing gear current injection configuration. On the right-hand side, the finite difference mesh

In this section we present an example of modelling of a current pulse injection in an aircraft landing gear (See Fig. 3). The finite-difference time-domain technique required several hours to obtain the field solution over some 2 micro seconds whereas the macro-modelling required only one minute to obtain the results upto 14 micro-seconds. Here, we do not count the time needed to construct the models themselves. But also constructing the finite difference discretisation needs a lot of effort whereas the macro-modelling can be done much faster and has the additional advantage that experts can easily adjust model parts "by hand". The simulation results show that the finite difference results quite correctly represent the current distribution over the various metallic parts of the landing gear. However, the results of the macromodelling are equally good and because the computation time is much shorter a better global view of the phenomena can be obtained in much less time.

\section{Conclusion}

In spite of the enormous power of present-day computing machines, dedicated modelling tools have advantages over generic tools based on universal discretisation methods. In the first place, it is important to recognise the distinction 

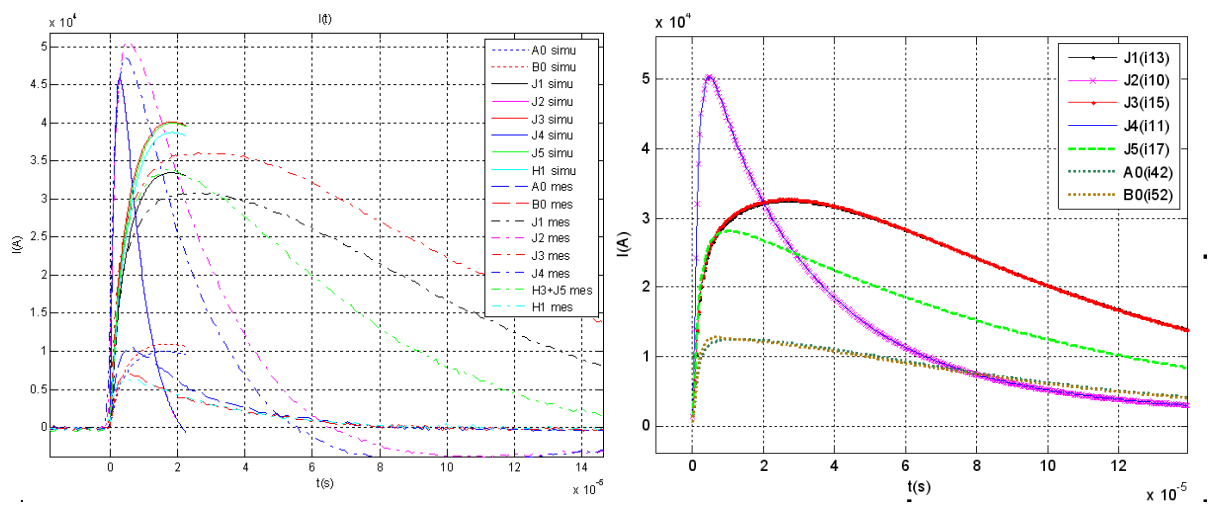

Fig. 4 Landing gear example comparisons

between the degrees of freedom needed in the geometric modelling and the degrees of freedom essential for the physical phenomena one is interested in. In addition, it is important to be able to influence directly and efficiently on the coefficients of the physical model. The macro-modelling described in this paper is an interesting candidate for lightning problems where one has to deal with large and complex geometries together with quasi-static current distributions of which the structure does not change very much during the process.

\section{References}

1. B.M. Maschke, A.J. van der Schaft and P.C. Breedveld, An Intrinsic Hamiltonian Formulation of the Dynamics of LC-Circuits, IEEE Trans. on Circuits and Systems, 42-I(2): pp. 73-82 (1995)

2. G. Antonini, D. Frigioni, G. Miscione: Hybrid Formulation of the Equation Systems of the 3-D PEEC Model Based on Graph Algorithms. IEEE Trans. on Circuits and Systems, 57-I(1): pp. 249-261 (2010)

3. F. P. Andriulli, F. Vipiana, and G. Vecchi. Hierarchical Bases for Nonhierarchic 3-D Triangular Meshes. IEEE TAP: 56-8, pp 2288-2298 (2008)

4. S. Adrian, T. Eibert, and F. P. Andriulli. Primal and Dual Graph Haar Bases for the Hierarchical Regularisation of the EFIE on Unstructured Meshes. International Conference on Electromagnetics in Advanced Applications (ICEAA), 2013

5. D. Prost, F.Issac, T. Volpert, W. Quenum and J.-P. Parmantier, Lightning-Induced Current Simulation Using RL Equivalent Circuit: Application to an Aircraft Subsystem Design IEEE Trans. EMC: 55-2, pp. 378-384 (2012) 